\title{
Vaccine schedules and the effect on humoral and intestinal immunity against poliovirus: a systematic review and network meta-analysis
}

\author{
Grace R Macklin, Nicholas C Grassly, Roland W Sutter, Ondrej Mach, Ananda S Bandyopadhyay, W John Edmunds, Kathleen M O'Reilly
}

\begin{abstract}
Summary
Background The eradication of wild and vaccine-derived poliovirus requires the global withdrawal of oral poliovirus vaccines (OPVs) and replacement with inactivated poliovirus vaccines (IPVs). The first phase of this effort was the withdrawal of the serotype 2 vaccine in April 2016, with a switch from trivalent OPVs to bivalent OPVs. The aim of our study was to produce comparative estimates of humoral and intestinal mucosal immunity associated with different routine immunisation schedules.
\end{abstract}

Methods We did a random-effect meta-analysis with single proportions and a network meta-analysis in a Bayesian framework to synthesise direct and indirect data. We searched MEDLINE and the Cochrane Library Central Register of Controlled Trials for randomised controlled trials published from Jan 1, 1980, to Nov 1, 2018, comparing poliovirus immunisation schedules in a primary series. Only trials done outside western Europe or North America and without variation in age schedules (ie, age at administration of the vaccine) between study groups were included in the analyses, because trials in high-income settings differ in vaccine immunogenicity and schedules from other settings and to ensure consistency within the network of trials. Data were extracted directly from the published reports. We assessed seroconversion against poliovirus serotypes 1,2 , and 3, and intestinal immunity against serotype 2, measured by absence of shedding poliovirus after a challenge OPV dose.

Findings We identified 437 unique studies; of them, 17 studies with a maximum of 8279 evaluable infants were eligible for assessment of humoral immunity, and eight studies with 4254 infants were eligible for intestinal immunity. There was low between-trial heterogeneity in the data $(\tau=0 \cdot 05,95 \%$ credible interval $[\mathrm{CrI}] \mathbf{0} 009-0 \cdot 15)$. For serotype 2, the risk ratio (RR) of seroconversion after three doses of bivalent OPVs was $0 \cdot 14(95 \% \mathrm{CrI} 0 \cdot 11-0 \cdot 17)$ compared with three doses of trivalent OPVs. The addition of one or two full doses of an IPV after a bivalent OPV schedule increased the RR to $0 \cdot 85(0 \cdot 75-1 \cdot 0)$ and $1 \cdot 1(0 \cdot 98-1 \cdot 4)$. However, the addition of an IPV to bivalent OPV schedules did not significantly increase intestinal immunity $(0 \cdot 33,0 \cdot 18-0 \cdot 61)$, compared with trivalent OPVs alone. For serotypes 1 and 3, there was susbstantial inconsistency and between-trial heterogeneity between direct and indirect effects, so we only present pooled estmates on seroconversion, which were at least $80 \%$ for serotype 1 and at least $88 \%$ for serotype 3 for all vaccine schedules.

Interpretation For WHO's polio eradication programme, the addition of one IPV dose for all birth cohorts should be prioritised to protect against paralysis caused by type 2 poliovirus; however, this inclusion will not prevent transmission or circulation in areas with faecal-oral transmission.

Funding UK Medical Research Council.

Copyright Copyright (C) 2019 The Author(s). Published by Elsevier Ltd. This is an Open Access article under the CC BY 4.0 license.

\section{Introduction}

In 1988, the World Health Assembly passed a resolution that committed WHO to eradicating poliomyelitis globally. The eradication effort has been centred on mass vaccination campaigns and achieving high routine immunisation coverage with Sabin oral poliovirus vaccines (OPVs) and Salk inactivated poliovirus vaccines (IPVs). ${ }^{1,2}$ More than 150 countries have relied on OPVs to eliminate poliovirus transmission and maintain a polio-free status; however, the cessation of all OPV use and replacement by IPVS is necessary because of the risk of vaccine-derived poliovirus and vaccine-associated paralytic poliomyelitis associated with the OPV. ${ }^{3}$ The first phase of this cessation was completed in April 2016, with the global withdrawal of the type 2 strain OPV and a switch from the trivalent OPV (tOPV) to a bivalent OPV (bOPV) formulation. Additionally, the Strategic Advisory Group of Experts on Immunisation (SAGE) recommended that at least one dose of IPV was introduced into all routine immunisation schedules to protect against poliomyelitis caused by serotype $2 .{ }^{4}$ However, constraints on IPV supply resulted in 39 countries having to delay IPV introduction or interrupt its routine use, with some countries adopting the use of intradermal fractional-dose IPV (fIPV). ${ }^{5}$
Lancet Infect Dis 2019 Published Online July 23, 2019 http://dx.doi.org/10.1016 S1473-3099(19)30301-9 See Online/Comment http://dx.doi.org/10.1016/ S1473-3099(19)30322-6

Department of Disease Control (G R Macklin MRes, K M O'Reilly PhD), Centre for Mathematical Modelling of Infectious Disease (G R Macklin Prof WJ Edmunds PhD, K M O'Reilly), and Department of Infectious Disease Epidemiology (Prof WJ Edmunds), London School of Hygiene \& Tropical Medicine, London, UK; Department of Infectious Disease Epidemiology, St Mary's Campus, Imperial College London, London, UK (Prof N C Grassly PhD); Polio Eradication Department, World Health Organization, Geneva, Switzerland (GR Macklin, RW Sutter MD, O Mach MD); and Bill \& Melinda Gates Foundation, Seattle, WA, USA (A S Bandyopadhyay MBBS) Correspondence to: Grace R Macklin, Department of Disease Control, London School of Hygiene \& Tropical Medicine, London WC1E 7HT, UK grace-ruth.macklin@Ishtm. ac.uk 
Research in context

\section{Evidence before this study}

The phased removal of the oral polio vaccine (OPV) is occurring alongside introduction of the inactivated poliovirus vaccine (IPV), eradication of wild poliomyelitis cases, and prevention of the emergence and circulation of vaccine-derived polioviruses. We searched MEDLINE and the Cochrane Library Central Register of Controlled Trials for randomised controlled trials published from Jan 1, 1980, to Nov 1, 2018, that compare poliovirus immunisation schedules in primary series to compile in a network meta-analysis. We used the search terms: (polio OR poliovirus) AND vaccine $A N D$ (primary series $O R$ routine $O R$ infants) AND (seropositive OR seroconversion OR antibody OR mucosal immunity OR intestinal immunity). Only trials done outside western Europe or North America and without variation in age schedules between study groups were included in the analyses. We assessed the risk of bais with the Cochrane Collaboration's tool for assessing bias in randomised trials and found it to be low-to-moderate for individual studies. The effect of various vaccine schedules on humoral and mucosal immunity has been addressed by many reviews and phase 3 clinical trials in different settings globally, creating a vast pool of recommendations for appropriate vaccine schedules.

\section{Added value of this study}

Schedules of bivalent OPV plus IPV have the potential to

provide humoral immunity against serotype 2 poliovirus, which protects against paralysis from circulating vaccine-derived poliovirus of serotype 2 . However, such a schedule will not induce intestinal protection against serotype 2, enabling transmission of this virus in some populations. There is little difference between the immunogenicity achieved through affordable IPV options (Salk IPV, Sabin IPV, fractional IPV, adjuvanted IPV, and monovalent IPV).

\section{Implications of all the available evidence}

Schedules of bivalent OPV plus IPV are recommended for routine immunisation in low-income and middle-income settings. Any vaccination programme must ensure that every child receives at least one dose of IPV to protect against poliomyelitis, which will require strong routine immunisation systems and catch-up of missed children. The interruption of all transmission of serotype 2 needs to be expedited and surveillance systems to detect circulating virus quickly need to be strengthened. Evidence supports the recommendations given by Strategic Advisory Group of Experts for the introduction of IPVs and the adoption of dose-sparing options, such as fractional IPV in times of IPV supply shortage.
After termination of OPV use, the post-eradication schedule is planned to comprise a minimum of two IPV doses given after 14 weeks of age. ${ }^{6}$ There are several approaches to developing affordable IPV options, including restricting the number of IPV doses in routine immunisation to two, reducing the volume of each dose through intradermal administration, reducing the antigen content of each dose through use of adjuvants, and reducing the cost of production through developing IPVs from attenuated vaccines of the Sabin strains of poliovirus. ${ }^{78}$ These approaches have resulted in the development of alternative formulations to conventional intramuscular Salk IPV, including intradermal fIPV, adjuvanted IPV, monovalent type 2 IPV (mIPV2), and Sabin IPV (sIPV).

Accordingly, many clinical trials have evaluated the immunogenicity of different vaccine schedules. It is essential to develop a comprehensive overview of immunity induced by different routine immunisation schedules against the three poliovirus serotypes. Standard meta-analysis approaches combine information from multiple studies to estimate the overall effectiveness of an intervention, but do not compare effectiveness between interventions that have not been explicitly trialled. By contrast, network meta-analysis uses the quantitative relatedness (ie, relative effects) of interventions to estimate both the direct and indirect effects. ${ }^{9,10}$ Although network meta-analyses are used increasingly to compare drugs, they have not been widely adopted to compare vaccine schedules. . $11,12^{2}$

In this Article, we aim to estimate the relative immunogenicity of the different OPV and IPV routine immunisation schedules considered by WHO and member states in inducing humoral and intestinal immunity against poliovirus. This knowledge would be useful to inform global immunisation policy.

\section{Methods}

\section{Search strategy and selection criteria}

We did a systematic review and network meta-analysis of randomised controlled trials comparing the immunogenicity of primary immunisation schedules for poliovirus vaccines in healthy infants and providing efficacy outcomes of the vaccination. Interventions of IPV-only, IPV-bOPV combination, and bOPV-only vaccine schedules were included, in comparison with each other or with a tOPV-only schedule. Interventions were included if the age of administration of the first vaccine dose (excluding a dose at birth) was between 4 and 8 weeks of age. A full study protocol outlining the population, intervention, comparison, and outcome criteria used is available in the appendix (p 3).

We searched MEDLINE and Cochrane Library Central Register of Controlled Trials (CENTRAL) for randomised controlled trials published from Jan 1, 1980, to Nov 1, 2018, using the search terms: (polio OR poliovirus) AND 
vaccine AND (primary series OR routine OR infants) AND (seropositive OR seroconversion OR antibody OR mucosal immunity OR intestinal immunity). Trials were excluded if they were done in western Europe or North America, because of differences in vaccine immunogenicity and schedules used in these highincome settings, or if there was variation in age schedules (ie, age at administration of the vaccine) between study groups, to ensure consistency within the network of trials we analysed. The most relevant or inclusive data (ie, fitting the search criteria and most standard procedures) for a given study, with no differentiation between vaccine manufacturer, were chosen. We follow the Preferred Reporting Items for Systematic Reviews and MetaAnalyses (PRISMA) guidelines to report our network meta-analysis.

We curated the retrieved studies on the basis of two predefined outcomes. The first outcome was seroconversion against poliovirus serotypes 1, 2, and 3, measured 4 weeks after the most recent vaccine dose. Seroconversion was defined as a change in antibody titre from non-detectable $(<1: 8)$ to detectable $(\geq 1: 8)$ antibody titre, or four-fold or higher increase in antibody titre over the expected decline of maternally derived antibodies (assuming a maternal antibody half-life of 28 days). ${ }^{13}$ The second outcome was development of intestinal immunity against serotype 2 . Immunity was measured as the absence of shedding of type 2 poliovirus 7 days after a challenge dose of OPV containing the Sabin type 2 strain.

The studies were reviewed and the data extracted independently by two of the investigators (GM and KMO'R). The number of individuals in each study group was recorded by serotype and time of sample collection. We also extracted data for study location, age at administration, route of administration, vaccine antigen content, and challenge vaccine and timing (where applicable). We assessed the risk of bias in accordance with the Cochrane Collaboration's tool ${ }^{14}$ for assessing risk of bias in randomised trials, for individual elements from five domains (selection, performance, attrition, reporting, and other bias) and the overall quality of evidence using the Grading of Recommendations Assessment, Development, and Evaluation framework..$^{15}$

\section{Data analysis}

We did a random-effect meta-analysis of single proportions, using an inverse variance pooling method and logit transformation, in the meta package in $\mathrm{R}$ (version 3.4.3). A random-effect network meta-analysis was developed for each outcome, with a binomial likelihood and log-link function and computed in a Bayesian framework using the GeMTC package in $\mathrm{R}$ (version 3.4.3). Markov chain Monte Carlo (MCMC) simulations estimated posterior distributions of relative treatment effects and SDs, with vague uniform priors. Four independent Markov chains were run with 10000 burn-in iterations and

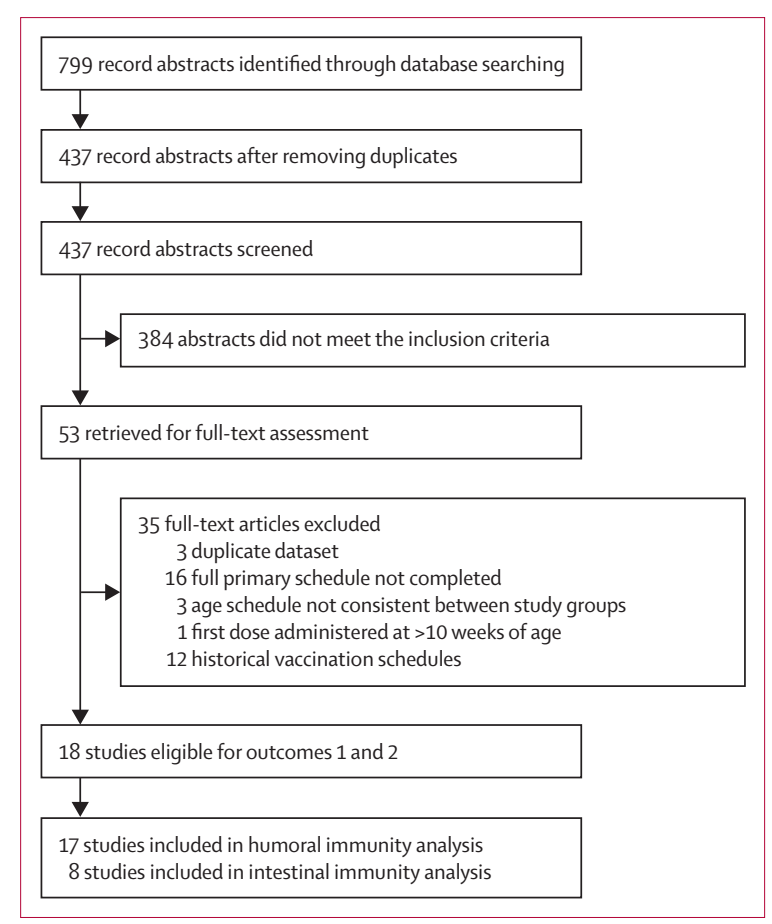

Figure 1: Systematic review profile

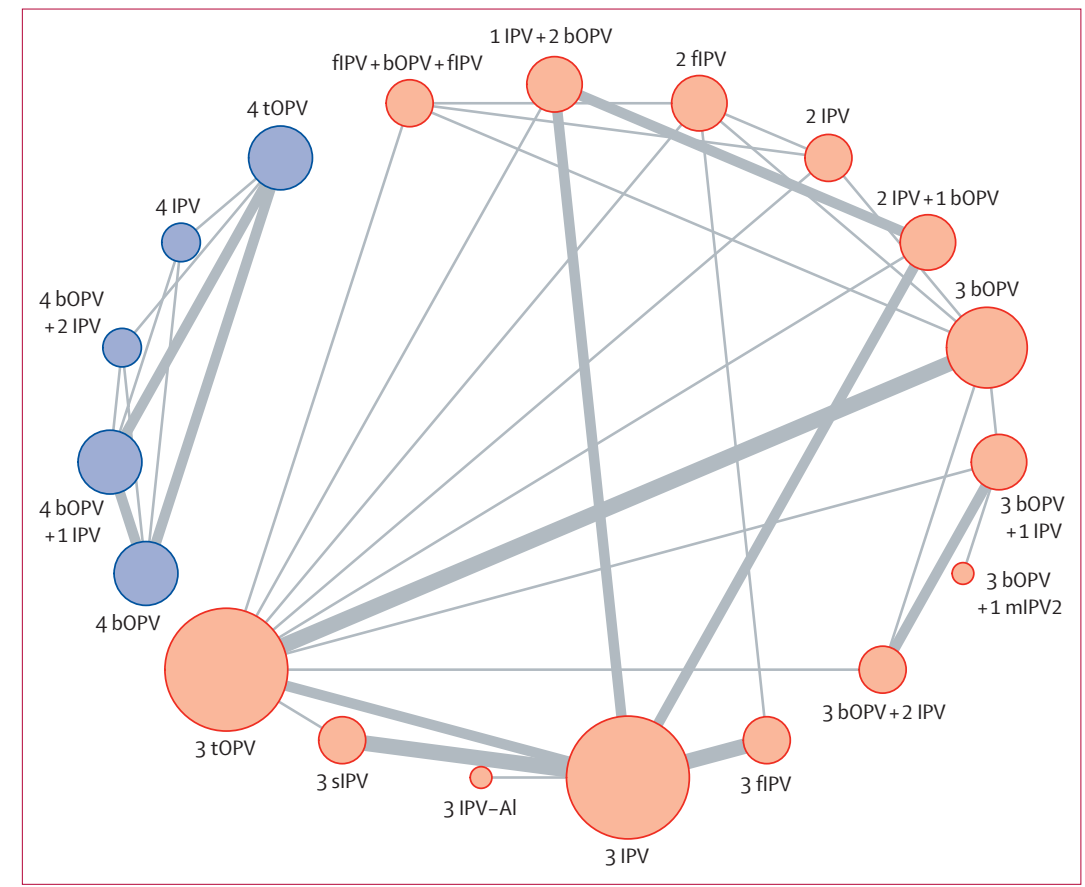

Figure 2: Networks of eligible comparisons for vaccination schedules inducing humoral immunity against poliovirus serotypes 1,2 , and 3

Each node represents a vaccine schedule and the lines are direct comparisons. The width of the lines is proportional to the number of trials compared and the node size is proportional to the number of trials that include that schedule. Blue schedules include a birth dose and orange schedules do not. bOPV=bivalent oral poliovirus vaccine. $\mathrm{fIPV}=$ fractional inactivated poliovirus vaccine. IPV=conventional inactivated poliovirus vaccine. IPV-Al=aluminium hydroxide adjuvanted inactivated poliovirus vaccine. $\mathrm{mIPV} 2=$ monovalent serotype 2 high-dose inactivated poliovirus vaccine. sIPV=Sabin inactivated poliovirus vaccine. $\mathrm{tOPV=trivalent} \mathrm{oral} \mathrm{poliovirus} \mathrm{vaccine.}$ 


\begin{tabular}{|c|c|}
\hline & Age schedule (weeks) \\
\hline$I P V+2$ bOPV & $8,12,16$ or $8,16,24$ \\
\hline $2 \mathrm{flPV}^{*}$ & 6,14 \\
\hline $2 \mathrm{IPV}$ & 6,14 \\
\hline $2 I P V+b O P V$ & $8,12,16$ or $8,16,24$ \\
\hline 3 bOPV & $6,10,14$ \\
\hline 3 bOPV + IPV & $6,10,14,14$ \\
\hline $3 \mathrm{bOPV}+\mathrm{mIPV} 2 \dagger$ & $6,10,14,14$ \\
\hline $3 \mathrm{bOPV}+2 \mathrm{IPV}$ & $6,10,14,14,36$ \\
\hline $3 \mathrm{fIPV}^{*}$ & $6,10,14$ or $8,16,24$ \\
\hline 3 IPV & $6,10,14 ; 8,12,16 ;$ or $8,16,24$ \\
\hline 3 IPV-Al $\neq$ & $6,10,14$ \\
\hline 3 sIPVS & $8,12,16$ \\
\hline 3 tOPV & $6,10,14$ or $8,12,16$ \\
\hline 4 bOPV & Birth, 6, 10, 14 \\
\hline 4 bOPV + IPV & Birth, $6,10,14,14$ \\
\hline $4 \mathrm{bOPV}+2 \mathrm{IPV}$ & Birth, 6, 10, 14, 18 \\
\hline $4 \mathrm{IPV}$ & Birth, 6, 10, 14 \\
\hline 4 tOPV & Birth, $4,8,12$ or birth, $6,10,14$ \\
\hline$f I P V^{*}+b O P V+f I P V^{*}$ & $6,10,14$ \\
\hline \multicolumn{2}{|c|}{$\begin{array}{l}\text { bOPV=bivalent oral poliovirus vaccine. fIPV=fractional inactivated poliovirus vaccine. } \\
\text { IPV=conventional inactivated poliovirus vaccine. IPV-Al=aluminium hydroxide } \\
\text { adjuvanted inactivated poliovirus vaccine. mIPV2=monovalent serotype } 2 \text { high-dose } \\
\text { inactivated poliovirus vaccine. sIPV=Sabin inactivated poliovirus vaccine. } \\
\text { tOPV=trivalent oral poliovirus vaccine. *One-fifth full IPV dose administered } \\
\text { intradermally. †32 D-antigen content for serotype } 2 \text { administered intramuscularly. } \\
\text { ‡One-tenth full IPV dose administered intramuscularly. } \$ 30,32 \text {, and } 45 \text { D-antigen } \\
\text { content for serotype 1, 2, and 3, respectively, administered intramuscularly. }\end{array}$} \\
\hline $\begin{array}{l}\text { Table: Vaccine schedu } \\
\text { for each node of the }\end{array}$ & $\begin{array}{l}\text { oximate age of administration included } \\
\text { ta-analysis for humoral immunity }\end{array}$ \\
\hline
\end{tabular}

60000 inference iterations per chain. Convergence of Markov chains was evaluated using the Gelman-RubinBrooke diagnostic and time-series plots. ${ }^{16}$ Autocorrelation plots were assessed to detect autocorrelation in the chains. Additional analysis included network metaregression to investigate the effect of study-level covariates, including the estimated mortality rate for children younger than 5 years due to diarrhoeal disease in the country of study location. ${ }^{17}$

We report the pooled random-effect estimates for singleproportion meta-analyses by study arm with $95 \%$ CIs. For the network meta-analysis, the between-intervention relative effects were summarised as risk ratios (RR) and reported as the median of the posterior distribution with 95\% credible intervals (CrIs). Differences between treatments are considered significant (at the 5\% level) if CIs do not overlap the no-effect line. The RRs are presented relative to a tOPV comparator and as relativeeffect tables between treatments (appendix p 21). Pairwise analysis is additionally reported for programmatically important comparisons (appendix p 27).

Model-fit was measured by deviance information criterion, residual deviance, and leverage. ${ }^{18}$ We show the SD of the random-effects model (known as $\tau$ ), as a measure of heterogeneity in the network, where $\tau^{2}$ is the between-study variance of the true effect size. A node-splitting model was generated to assess inconsistency within the network. ${ }^{10}$

\section{Role of the funding source}

The funders had no role in study design, data collection, data analysis, data interpretation, or writing of the report. The corresponding author had full access to all the data in the study and had final responsibility for the decision to submit for publication.

\section{Results}

A literature search in MEDLINE and CENTRAL identified 437 unique studies and 53 were retrieved for full-text assessment (figure 1). 17 studies describing 47 study groups met the inclusion criteria and were included in the network meta-analysis for humoral immunity, and eight studies describing 25 study groups did so for intestinal immunity (appendix p 6-14). We found a low-to-moderate risk of bias for individual studies and a moderate-to-high quality of evidence for each outcome (appendix p 14). Seven vaccine formulations were included in the total analysis: tOPV, bOPV, Salk IPV (referred to as IPV and administered intramuscularly), mIPV2 (administered intramuscularly), fIPV (one-fifth Salk IPV dose, administered intradermally), sIPV (administered intramuscularly), and aluminium-adjuvanted IPV (IPV-Al; one-tenth Salk IPV dose, administered intramuscularly).

There were 19 unique vaccination schedules identified, and each formed a node in the network (figure 2) with corresponding schedules by patient age (table). The network was separated into two subgroups, studies without a birth dose (14 nodes, 15 studies) and those with a birth dose (five nodes, two studies). In total, 8254 infants were included in the analysis for seroconversion against serotype 1, 8241 against serotype 2, and 8279 against serotype 3 . The pooled proportion for all three serotypes of individuals who underwent seroconversion ranged from $13 \%$ to $100 \%$ between different vaccine schedules.

In the network meta-analysis, the criteria for adequate model-fit were satisfied with no significant inconsistency between any direct and indirect estimates $(p<0.05$ for all comparisons) and low between-trial heterogeneity ( $\tau=0 \cdot 05,95 \%$ CrI 0.009-0 15; appendix p 18).

All vaccine schedules achieved higher seroconversion RRs against serotype 2 than bOPV alone (figure 3). The addition of a single dose of IPV to a three-dose bOPV schedule significantly improved seroconversion (at age 18 weeks; RR $0 \cdot 85$, 95\% CrI $0 \cdot 75-1 \cdot 0$ ), and a second incremental increase in RR was seen after a second dose of IPV (at 36 weeks; 1.1, 0.98-1.4). A pairwise analysis of studies that directly compared seroconversion from three bOPV doses plus two IPV doses with three bOPV doses plus one IPV doses gave a pooled RR of $1 \cdot 25$ (95\% CrI 1.07-1.47; appendix p 27).

Combined bOPV-IPV schedules where IPV administration preceded bOPV had a lower RR than in cases in which IPV succeeded bOPV (figure 3). 


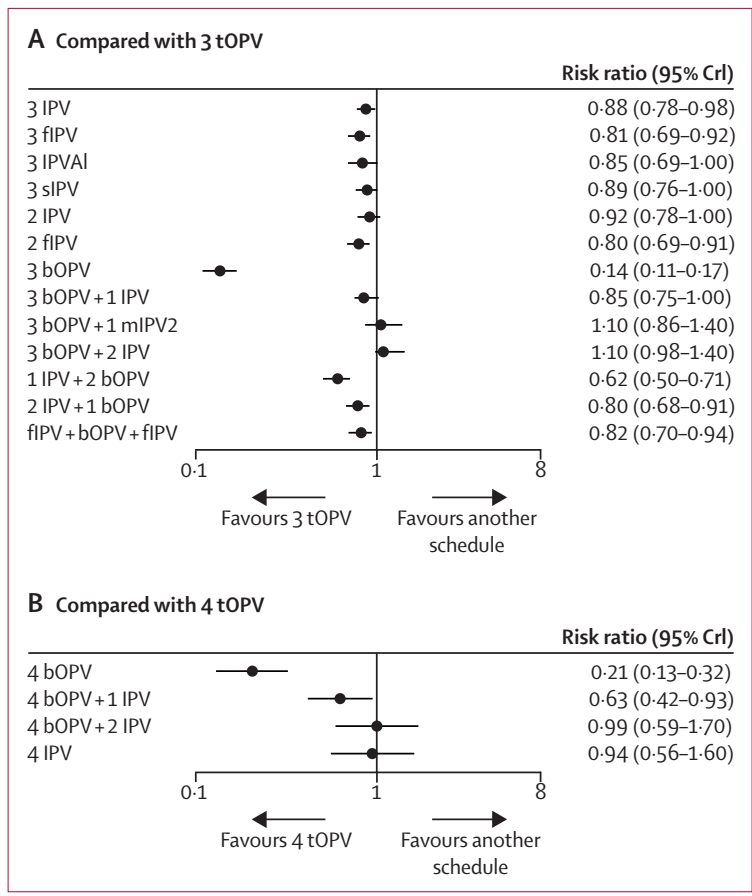

Figure 3: Relative immunogenicity of vaccine schedules for seroconversion against poliovirus serotype 2 schedules with no birth dose and schedules with a birth dose

Between-trial heterogeneity for $(A)$ schedules without a birth dose $(\tau=0.05$, 95\% $\mathrm{Crl} 0.009-0.15)$ and (B) those with a birth dose $(0.23,0.026-1.31)$. bOPV=bivalent oral poliovirus vaccine. $\mathrm{Crl}=$ credible interval. $\mathrm{flPV}=$ fractional inactivated poliovirus vaccine administered intradermally (one-fifth dose of IPV). $\mathrm{IPV}=$ conventional inactivated poliovirus vaccine. IPV-Al=aluminium hydroxide adjuvanted inactivated poliovirus vaccine (one-tenth reduced dose of IPV). $\mathrm{mIPV} 2=$ monovalent serotype 2 high-dose inactivated poliovirus vaccine. $S I P V=S a b i n$ inactivated poliovirus vaccine. $t O P V=$ trivalent oral poliovirus vaccine.

We compared IPV-only schedules for both two and three doses (see appendix p 21 for a table of relative effects). There was no significant difference between the seroconversion achieved by two doses of full-dose IPV and intradermal fIPV (RR 0 88 , 95\% CrI 0.74-1·02). Adding a third dose to the schedule gave no significant increase in seroconversion: the RR was $0.96(0 \cdot 81-1 \cdot 15)$ for two versus three doses of full-dose IPV, and $1 \cdot 01(0 \cdot 85-1 \cdot 20)$ for two versus three doses of intradermal fIPV. Additionally, there was no significant difference between three doses of any alternative IPV formulation with Salk IPV, fIPV $(0 \cdot 92,0 \cdot 83-1 \cdot 0)$, sIPV $(1 \cdot 01,0 \cdot 93-1 \cdot 10)$, or IPV-Al $(0 \cdot 96,0 \cdot 83-1 \cdot 11)$.

For schedules including a birth dose from the full network analysis, the addition of one or two doses of IPV following a four-dose bOPV-only schedule increased seroconversion (figure 3).

For the network meta-analysis of serotype 1, there was significant inconsistency between direct and indirect effects in one network comparison, and in six comparisons for serotype 3 . There was high between-trial heterogeneity for both serotypes (serotype $1 \tau=0 \cdot 24, \quad 95 \% \mathrm{CrI}$ $0 \cdot 12-0 \cdot 48$; serotype $3 \tau=0 \cdot 17,0 \cdot 09-0 \cdot 32$; appendix p 20). Therefore, we present the results of the individual trial

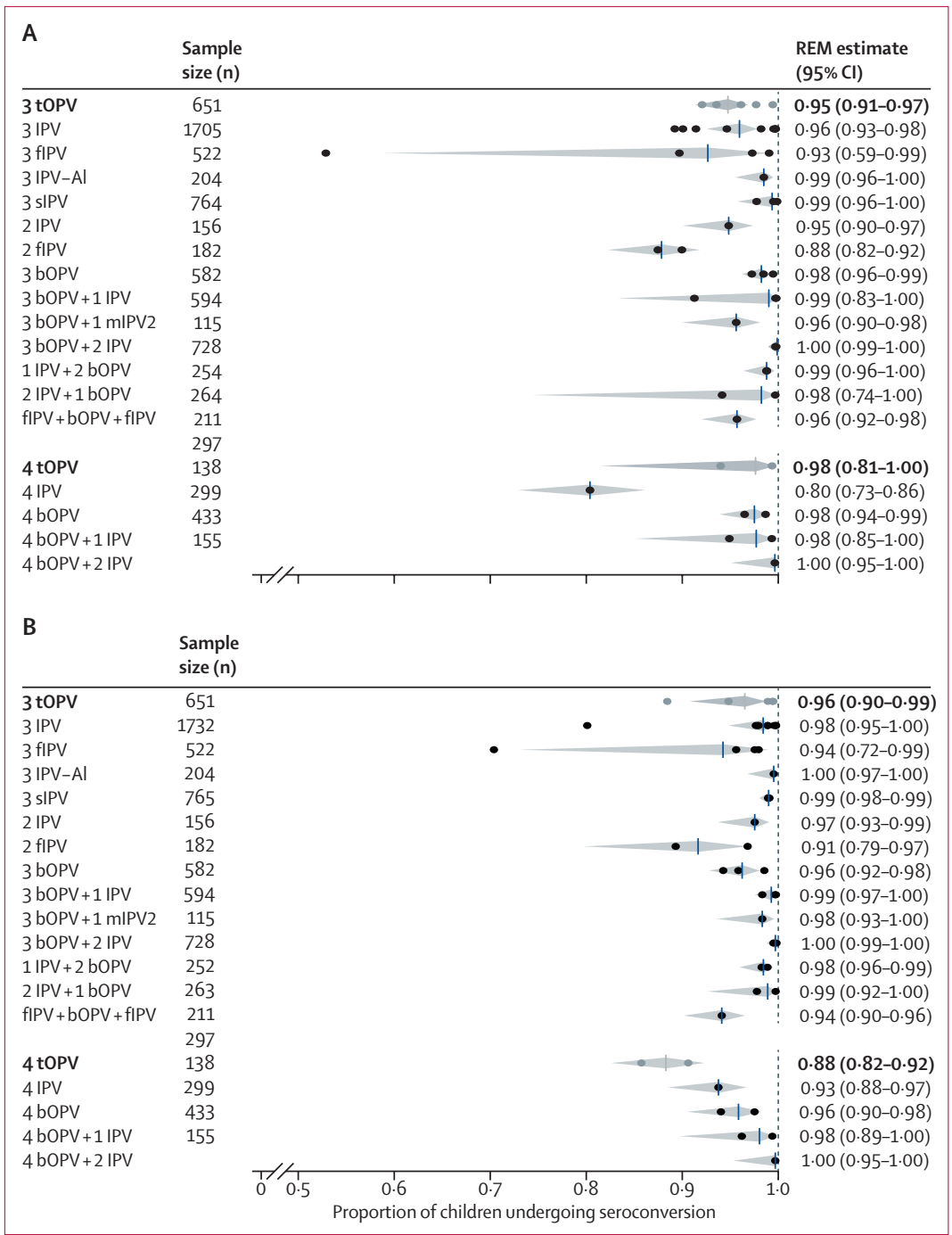

Figure 4: Random-effect single-proportion meta-analysis estimate of the proportion of children undergoing seroconversion against serotypes 1 (A) and 3 (B)

Overall REM estimates and heterogeneity were 0.97 (95\% $\mathrm{Crl} 0.96-0.98)$ and $\tau^{2}=1.66$ for serotype 1 and $0.97(0.96-0.98)$ and $\tau$-squared $=1.38$ for serotype 3 . Individual studies are shown as dots, with the overall estimated proportion as a vertical line through each dot and $95 \% \mathrm{Crl}$ as shading. bOPV=bivalent oral poliovirus vaccine. $\mathrm{Crl}=$ credible interval. $\mathrm{flPV}=$ fractional inactivated poliovirus vaccine administered intradermally (one-fifth dose of IPV). IPV=conventional inactivated poliovirus vaccine. IPV-AI=Aluminium hydroxide adjuvanted inactivated poliovirus vaccine (one-tenth reduced dose of IPV). $\mathrm{mIPV} 2=$ monovalent serotype 2 high-dose inactivated poliovirus vaccine. $\mathrm{REM}=$ random effect model. sIPV=Sabin inactivated poliovirus vaccine. tOPV=trivalent oral poliovirus vaccine

data and pooled estimates only: all vaccine schedules had pooled estimates for seroconversion of at least $80 \%$ for serotype 1 and at least $88 \%$ for serotype 3 (figure 4). For both serotypes, three doses of bOPV alone gave high seroconversion (figure 4). Additionally, there was little difference in seroconversion between three doses of IPV, fIPV, sIPV or IPV-Al, or between two doses of IPV and fIPV (figure 4).

There were 15 unique vaccination schedules identified for intestinal immunity against serotype 2 (see appendix $\mathrm{p}$ 29 for the single-proportion meta-analysis). The average 


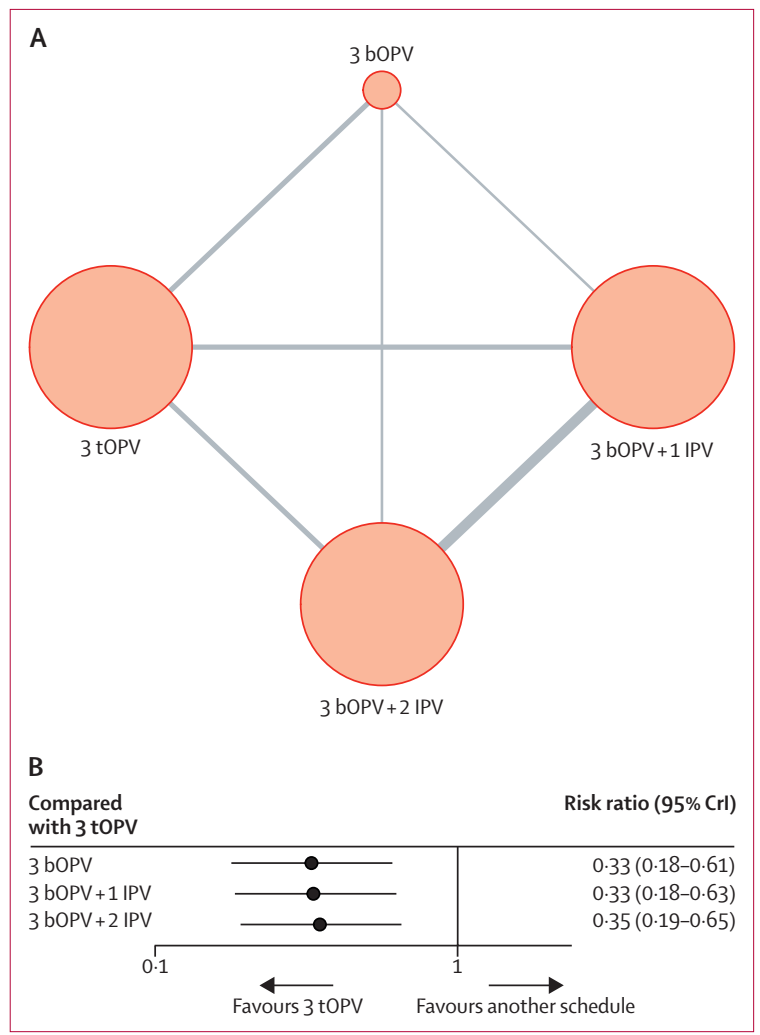

Figure 5: Network meta-analysis of routine immunisation schedules for intestinal immunity against serotype 2

(A) Network plot. Each node represents a vaccine schedule and the lines are direct comparisons. The width of the lines is proportional to the number of trials compared and the node size is proportional to the number of trials that include that schedule. (B) Relative risk of absence of shedding vaccine-derived poliovirus after challenge, compared with three tOPV doses. Data are risk ratio $(95 \% \mathrm{Crl})$. bOPV=bivalent oral poliovirus vaccine. $\mathrm{Cr}=$ =credible interval. IPV=conventional inactivated poliovirus vaccine. $\mathrm{tOPV}=$ trivalent oral poliovirus vaccine.

proportion of individuals who developed intestinal immunity was $0.91 \quad(95 \%$ CI $0.70-0 \cdot 98)$ following three tOPV doses, $0 \cdot 30(0 \cdot 17-0 \cdot 48)$ following three bOPV doses, $0 \cdot 25(0 \cdot 22-0 \cdot 29)$ following three bOPV doses plus one IPV dose, and $0 \cdot 28(0 \cdot 22-0 \cdot 29)$ following three bOPV doses plus two IPV doses. Heterogeneity was high between studies, and was significant for schedules of three tOPV doses $(\tau=1 \cdot 56, p<0 \cdot 01)$, three bOPV doses $(\tau=0 \cdot 53, \mathrm{p}<0 \cdot 01)$, and four bOPV doses plus one IPV dose $(\tau=0 \cdot 77, \mathrm{p}<0 \cdot 01)$.

Only four routine immunisation schedules had data from multiple studies: three tOPV, three bOPV, three bOPV plus one IPV, and three bOPV plus two IPV doses. Therefore, another network was generated out of these four nodes, which was directed at a programmatic question of the added benefit of IPV to intestinal immunity (figure 5). Intestinal immunity was significantly lower for all schedules than three doses of tOPV. The addition of one or two doses of IPV to three doses of bOPV did not substantially alter the risk (figure 5).

\section{Discussion}

To our knowledge, we report the first application of a network meta-analysis to assess the immunogenicity of vaccine schedules against poliomyelitis and provide a single, comprehensive analysis of polio routine immunisation schedules for humoral and intestinal outcomes. We found that for humoral immunity the addition of one dose of IPV to bOPV schedules increases the immunity against serotype type 2 , that there is no difference in relative immunogenicity of IPV variants (Salk IPV, SIPV, intradermal fIPV, IPV-Al), and that the timing of the IPV dose in bOPV-IPV schedules is associated with immunogenicity. Potentially, the most important finding of our study is for mucosal immunity, as we found no evidence of increased intestinal immunity against serotype 2 associated with an addition of IPV to a bOPVonly schedule.

Clinical trials have provided valuable information to inform policy but are limited by specific head-to-head schedule comparisons, small cohort sizes, and generation of country-specific data. Previous literature reviews and meta-analyses have addressed specific aspects of vaccination against poliomyelitis virus: mucosal immunity induced by OPV versus IPV, ${ }^{19}$ humoral immunity of one versus two doses of IPV,,$^{20}$ and immunogenicity of two doses of fIPV. ${ }^{21}$ Additionaly, reviews and metaanalysis have compared the immunogenicity of bOPVIPV mixed schedules and IPV alone. ${ }^{22,23}$ In agreement with our results, a meta-analysis by Tang and colleagues ${ }^{23}$ found no significant difference between IPV-only and IPV-OPV schedules in seroconversion against serotypes 1 and 3. However, they included only six trials with two different schedule groups (IPV-only and IPV-OPV mixed schedule) and provided no data on mucosal immunity. Therefore, our analysis goes beyond what previous studies have done.

After the switch from tOPV to bOPV, SAGE recommended the introduction of at least one dose of IPV at age 14 weeks or older to provide an immunity base to type 2 poliovirus. Our results provided evidence that individuals vaccinated with bOPV-only schedules have negligible immunity against poliovirus 2 (probably from passive type- 2 exposure or antibody cross-neutralisation from types 1 and 3). This immunity deficit suggests that the estimated 43 million children across 33 countries who did not recieve IPV because of supply shortages have no protection..$^{24}$ Notably, the addition of a single dose of IPV (at 14 weeks) improved humoral immunity against serotype 2 , whereas a second dose (at 18 or 36 weeks) had a smaller impact and a single mIPV2 dose provided equivalent immunogenicity to two doses of trivalent IPV. Our results also highlight that the order and timing of the IPV dose in mixed schedules is important, with a reduced immunogenicity against the type 2 strain in cases where IPV preceded bOPV. This effect is likely due to the earlier age at which IPV is administered and the effect of maternal antibodies. ${ }^{25}$ 
Of note, the addition of IPV had a negligible effect on the development of intestinal immunity against serotype 2 . Although the ability of IPV to induce humoral immunity is established, IPV has a more complex role in mucosal immunity. IPV-only schedules provide inadequate intestinal immunity and do not prevent viral shedding following a challenge dose, but they might reduce the quantity and duration of shedding. ${ }^{26,27}$ However, IPV has been shown to boost mucosal protection in OPV-primed individuals. ${ }^{26}$ Our results provide evidence that the prime-boost model established for IPV works in a serotype-specific manner.

Our findings have several limitations. Consistency of the network is a fundamental assumption of network meta-analyses, ${ }^{9,10}$ which was not met for serotypes 1 and 3 for which heterogeneity and inconsistency persisted through the subgroup and regression analyses (appendix p 18-20). The type, schedule, and immunogenicity of poliovirus vaccines varies by location. ${ }^{3}$ The studies in this analysis $^{3}$ were done in eastern Mediterranean and Latin American countries that have primary vaccine schedules in which the first (non-birth) dose is administered between 4 and 8 weeks. Therefore, our results are primarily useful for policy makers in these settings. The geographical and age-schedule variation in absolute immunogenicity is incorporated into our study as the network meta-analysis method models the relative effects between vaccines, thus eliminating differences in baseline immunogenicity of comparator schedules. ${ }^{28}$

The most widely adopted measure of poliovirus mucosal immunity is through administration of a challenge dose of OPV and a subsequent collection of stool samples. Extrapolating intestinal immunity and transmission impact on the basis of absence of shedding 7 days following a challenge dose does not capture the duration of shedding, quanitity of virus shed, or nasopharengeal immunity. ${ }^{19,26}$ However, these meaures represent the best proxy for intestinal immunity to poliovirus available from clinical trial data. Finally, our analysis only provides estimates on protection within the timescale of the trials.

There are research gaps highlighted in our modelled networks, particularly the need for evaluation of mucosal immunity in more studies. A schedule of three bOPV doses followed by fIPV has not been included in randomised controlled trials, yet this schedule has been adopted in India and Sri Lanka. ${ }^{6}$ Research is needed to compare IPV-only vaccine schedules in a post-eradication setting and address the need to develop a live vaccine with improved genetic stability. A novel OPV, which is a live attenuated vaccine with a lower risk of reversion than the standard OPV, and an IPV plus dmLT adjuvant are being trialled for induction of mucosal immunity. ${ }^{6,29-31}$

The findings of our comprehensive analysis show that a network meta-analysis is effective to evaluate multi-arm vaccination studies. Our results support, with policy recommendations from the SAGE, the addition of IPV into routine immunisation schedules and the adoption of affordable IPV approaches. We show that a single dose of IPV improves humoral immunity against serotype 2 and suggest that in times of IPV supply constraints, equitable distribution of a single dose of IPV should be prioritised over cohorts receiving a second dose, taking into account country-specific risk. This IPV addition will be unlikely to prevent faecal-oral transmission of the virus, but would provide individual protection against paralytic disease.

\section{Contributors}

GRM, WJE, and RWS contributed to study concept and design. GRM and KMO'R independently reviewed studies and extracted data, did the statistical analysis, and drafted the report. GM, WJE, OM, RWS, NCG, ASB, and KMO'R contributed to critical revision of the manuscript for intellectual content.

\section{Declaration of interests}

We declare no competing interests.

\section{Acknowledgments}

The funding source for this research was through a UK Medical Research Council PhD studentship held by GRM. We thank the Polio Research Committee for their discussions that initially highlighted the importance of this research.

References

1 Sutter RW, Kew OM, Cochi SL, Aylward RB. Poliovirus vaccine-live. In: Plotkin SA, Orenstein WA, Offit PA, eds. Vaccines, 6th edn. Philadelphia, PA: Elsevier, 2013: 598-645.

2 Vidor E, Plotkin SA. Poliovirus-inactivated In: Plotkin SA, Orenstein WA, Offit PA, eds. Vaccines, 6th edn. Philadelphia, PA: Elsevier, 2013: 573-97.

3 Kew OM, Sutter RW, de Gourville EM, Dowdle WR, Pallansch MA. Vaccine-derived polioviruses and the endgame strategy for global polio eradication. Annu Rev Microbiol 2005; 59: 587-635.

4 Global Polio Eradication Initiative. Polio Eradication and Endgame Strategic Plan 2013-2018. Geneva: World Health Organization, 2013.

5 Hampton LM, Farrell M, Ramirez-Gonzalez A, et al. Cessation of trivalent oral poliovirus vaccine and introduction of inactivated poliovirus vaccine-worldwide, 2016. MMWR Morb Mortal Wkly Rep 2016; 65: 934-38.

6 Bahl S, Bhatnagar P, Sutter RW, Roesel S, Zaffran M. Global polio eradication-way ahead. Indian J Pediatr 2018; 85: 124-31.

7 Okayasu H, Sutter RW, Jafari HS, Takane M, Aylward RB. Affordable inactivated poliovirus vaccine: strategies and progress. J Infect Dis 2014; 210 (suppl 1): S459-64.

8 Bakker WA, Thomassen YE, van't Oever AG, et al. Inactivated polio vaccine development for technology transfer using attenuated Sabin poliovirus strains to shift from Salk-IPV to Sabin-IPV. Vaccine 2011; 29: 7188-96.

9 Lu G, Ades AE. Combination of direct and indirect evidence in mixed treatment comparisons. Stat Med 2004; 23: 3105-24.

10 van Valkenhoef G, Dias S, Ades AE, Welton NJ. Automated generation of node-splitting models for assessment of inconsistency in network meta-analysis. Res Synth Methods 2016; 7: 80-93.

11 McGirr A, Widenmaier R, Burnett H, et al. Network meta-analysis in the evaluation of vaccines. Value Health 2017; 20: A777.

12 Tricco AC, Zarin W, Cardoso R, et al. Efficacy, effectiveness, and safety of herpes zoster vaccines in adults aged 50 and older: systematic review and network meta-analysis. BMJ 2018; 363: k4029.

13 Cohenabbo A, Culley BS, Reed GW, et al. Seroresponse to trivalent oral poliovirus vaccine as a function of dosage interval. Pediatr Infect Dis J 1995; 14: 100-06.

14 Higgins JP, Altman DG, Gotzsche PC, et al. The Cochrane Collaboration's tool for assessing risk of bias in randomised trials. BMJ 2011; 343: d5928.

15 Guyatt GH, Oxman AD, Vist GE, et al. GRADE: and emerging consensus on rating quality of evidence and strength of recommendations. BMJ 2008; 336: 924-26.

16 Stephen PB, Gelman A. General methods for monitoring convergence of iterative simulations. J Comput Graph Stat 1998; 7: 434-55. 
Articles

17 GBD Diarrhoeal Diseases Collaborators. Estimates of global, regional, and national morbidity, mortality, and aetiologies of diarrhoeal diseases: a systematic analysis for the Global Burden of Disease Study 2015. Lancet Infect Dis 2017; 17: 909-48.

18 Spiegelhalter DJ, Best NG, Carlin BR, van der Linde A. Bayesian measures of model complexity and fit. J Roy Stat Soc B 2002; 64: 583-616.

19 Hird TR, Grassly NC. Systematic review of mucosal immunity induced by oral and inactivated poliovirus vaccines against virus shedding following oral poliovirus challenge. PLoS Pathog 2012; 8: e1002599.

20 Grassly NC. Immunogenicity and effectiveness of routine immunization with 1 or 2 doses of inactivated poliovirus vaccine: systematic review and meta-analysis. J Infect Dis 2014; 210: S439-46.

21 Anand A, Molodecky NA, Pallansch MA, Sutter RW. Immunogenicity to poliovirus type 2 following two doses of fractional intradermal inactivated poliovirus vaccine: a novel dose sparing immunization schedule. Vaccine 2017; 35: 2993-98.

22 Bandyopadhyay AS, Modlin JF, Wenger J, Gast C. Immunogenicity of new primary immunization schedules with inactivated poliovirus vaccine and bivalent oral polio vaccine for the polio endgame: a review. Clin Infect Dis 2018; 67 (suppl 1): S35-41.

23 Tang G, Yin W, Cao Y, et al. Immunogenicity of sequential inactivated and oral poliovirus vaccines (OPV) versus inactivated poliovirus vaccine (IPV) alone in healthy infants: a systematic review and meta-analysis. Hum Vaccin Immunother 2018; 9: 1-8.

24 WHO. 16th Meeting of the SAGE Polio Working

Group-conclusions and recommendations: note for the Record. Geneva: World Health Organization, 2018
25 Gaensbauer JT, Gast C, Bandyopadhyay AS, et al. Impact of maternal antibody on the immunogenicity of inactivated polio vaccine in infants immunized with bivalent oral polio vaccine: implications for the polio eradication endgame. Clin Infect Dis 2018 67 (suppl 1): S57-65.

26 Parker EP, Molodecky NA, Pons-Salort M, O’Reilly KM, Grassly NC. Impact of inactivated poliovirus vaccine on mucosal immunity: implications for the polio eradication endgame. Expert Rev Vaccines 2015; 14: 1113-23.

27 Sutter RW. Unraveling the mucosal immunity of inactivated poliovirus vaccine. J Infect Dis 2018; 217: 344-46.

28 van Valkenhoef G, Lu G, de Brock B, Hillege H, Ades AE, Welton NJ. Automating network meta-analysis. Res Synth Methods 2012; 3: 285-99.

29 Norton EB, Bauer DL, Weldon WC, Oberste MS, Lawson LB, Clements JD. The novel adjuvant dmLT promotes dose sparing mucosal immunity and longevity of antibody responses to the inactivated polio vaccine in a murine model. Vaccine 2015; 33: 1909-15.

30 Bandyopadhyay AS, Garon J, Seib K, Orenstein WA. Polio vaccination: past, present and future. Future Microbiol 2015; 10: 791-808.

31 Van Damme P, De Coster I, Bandyopadhyay AS, et al. The safety and immunogenicity of two novel live attenuated monovalent (serotype 2 ) oral poliovirus vaccines in healthy adults: a double-blind, single-centre phase 1 study. Lancet 2019; 394: 148-58. 